\title{
Beyond the $T_{1}$ Limit: Singlet Nuclear Spin States in Low Magnetic Fields
}

\author{
Marina Carravetta, Ole G. Johannessen, and Malcolm H. Levitt* \\ School of Chemistry, University of Southampton, SO17 1BJ, United Kingdom
}

(Received 15 November 2003; published 14 April 2004)

\begin{abstract}
Low-field nuclear spin singlet states may be used to store nuclear spin order in a room temperature liquid for a time much longer than the spin-lattice relaxation time constant $T_{1}$. The low-field nuclear spin singlets are unaffected by intramolecular dipole-dipole relaxation, which is generally the predominant relaxation mechanism. We demonstrate storage of nuclear spin order for more than 10 times longer than the measured value of $T_{1}$. This phenomenon may facilitate the development of nuclear spin hyperpolarization methods and may allow the study of motional processes which occur too slowly for existing NMR techniques. This is the first time that the memory of nuclear spins has been extended well beyond the $T_{1}$ limit in a system lacking intrinsic magnetic equivalence.
\end{abstract}

DOI: $10.1103 /$ PhysRevLett.92.153003

PACS numbers: $33.25 .+\mathrm{k}$

One of the principal factors contributing to the wideranging utility of nuclear magnetic resonance (NMR) is the relatively long lifetime of nuclear spin order. This allows NMR spectroscopists to follow processes such as diffusion, flow, and slow molecular motion [1,2], and to contemplate using NMR for quantum computation $[3,4]$. The relaxation of the nuclear spins back to thermal equilibrium, and hence the lifetime of the nuclear spin memory, is characterized by a time constant $T_{1}$ known as the longitudinal, or spin-lattice, relaxation time constant. In many cases, this parameter is of the order of seconds, although it may be much longer in special cases. Major changes in $T_{1}$ can usually only be achieved by changing the isotopic composition of the sample or by changing its physical state. The sophisticated manipulation of nuclear spin interactions by radio-frequency (rf) pulse sequences, so prevalent in modern NMR [1], has always been restricted to a time frame set by the intrinsic $T_{1}$ limit. For example, the study of nuclear cross relaxation, which is exploited in molecular structure studies [5], is impeded by competing $T_{1}$ relaxation. NMR studies of flow and diffusion are also $T_{1}$ limited. Experiments which seek to enhance NMR signals by hyperpolarization phenomena [6-10] have great potential in NMR spectroscopy and NMR imaging, but are hampered by the need to use the hyperpolarized substance within a time of the order of $T_{1}$. NMR quantum computing is also limited by the memory time $T_{1}$.

In this Letter we demonstrate the storage of nuclear spin order for a time much longer than $T_{1}$. So far, we have demonstrated this phenomenon on molecules containing pairs of inequivalent protons, in which the main mechanism for nuclear spin relaxation is the modulation of the intramolecular dipole-dipole coupling by molecular motion. The extension of nuclear spin memory beyond the $T_{1}$ limit is achieved by creating nuclear spin singlet states in low magnetic field ( $20 \mathrm{mT}$ or less). These states are antisymmetric with respect to spin exchange, and are unaffected by intramolecular dipole-dipole relaxation. The low-field singlet (LFS) population therefore relaxes with a time constant much longer than $T_{1}$. This is a clear experimental realization of the "noiseless subsystem" concept developed in the context of quantum computing [11-16].

The LFS states are generated by adiabatic transfer from nonequilibrium high-field states. The singlet states are allowed to decay in low field and then transferred adiabatically back to high field where they can be detected as perturbations of conventional NMR signals. LFS states have been observed to decay with a time constant $T_{\mathrm{LFS}}=104 \pm 5 \mathrm{~s}$, in a sample for which the measured $T_{1}$ is $16.8 \pm 0.3 \mathrm{~s}$ under identical conditions. To our knowledge, this is the first demonstration of nuclear spin order storage for significantly longer than $T_{1}$ in a system lacking intrinsic magnetic equivalence. This is also the first experimental implementation of a "noisefree subspace" [14] which is immune to realistic dipoledipole relaxation.

The experimental procedure for investigating the lifetime of LFS states is sketched in Fig. 1. In its simplest form, the experiment applies to an ensemble of molecules, each containing a pair of spin- $1 / 2$ of the same isotopic type, but with different chemical shifts $\delta_{1}$ and $\delta_{2}$, and a mutual scalar coupling $J$. The experimental procedure uses a physical transport of the sample between two different magnetic field strengths, $B_{\text {high }}$ and $B_{\text {low }}$, where $B_{\text {high }} \gg B_{\text {low }}$, as shown in Fig. 1(a). The transport processes from high to low field, and vice versa, take times $\tau_{\text {transp }}^{(1)}$ and $\tau_{\text {transp }}^{(2)}$, respectively, which are assumed to be less than $T_{1}$. The low-field storage interval $\tau_{\mathrm{LF}}$ may be much longer than $T_{1}$. The transport between the two magnetic fields is synchronized with two rf pulse sequences denoted $A_{ \pm}$and $B$ in Fig. 1(b), specified below, and applied at the Larmor frequency of the spins in the high magnetic field.

The energy level picture at time point (1) in Fig. 1(c) shows the four Zeeman energy levels, with energies in the order $E_{\alpha \alpha}<E_{\alpha \beta}<E_{\beta \alpha}<E_{\beta \beta}$, assuming that $\delta_{2}>\delta_{1}$, the gyromagnetic ratio $\gamma$ and the $J$ coupling are both positive, and that the spin system is weakly coupled in high field. 


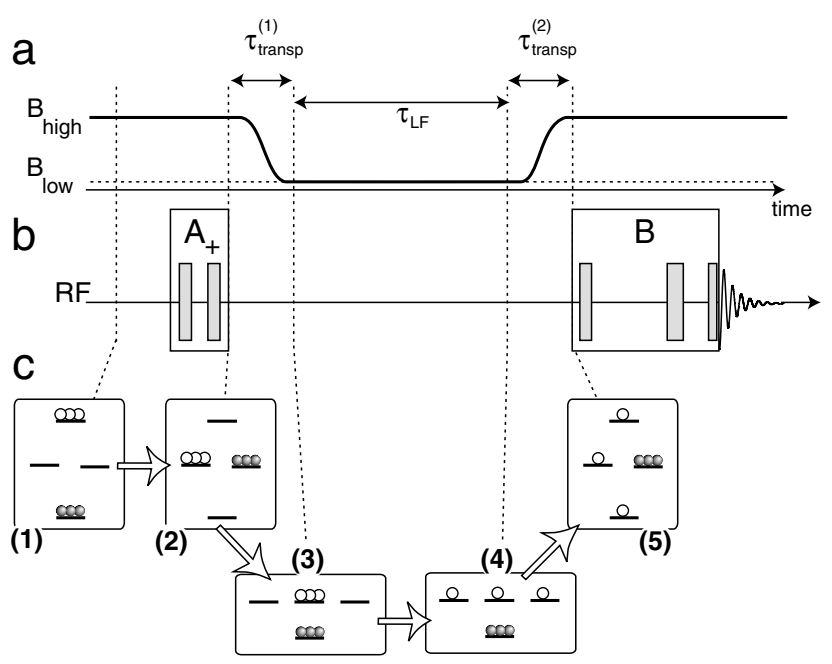

FIG. 1. (a) Sequence of magnetic fields used in the experiment. The sample is transported from a region of high magnetic field, $B_{\text {high }}$, into a region of low magnetic field, $B_{\text {low }}$, in a

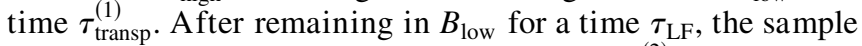
is transported back into $B_{\text {high }}$, in a time $\tau_{\text {transp }}^{(2)}$ (b) Pulse sequence performed at the Larmor frequency of the spins in high field. (c) Idealized sequence of spin state populations during the experiment. The four high-field energy levels belong to the states $|\beta \beta\rangle,|\alpha \beta\rangle,|\alpha \alpha\rangle$, and $|\beta \alpha\rangle$, reading in a clockwise direction starting with the upper state.

In thermal equilibrium at ambient temperature, the lowermost level $|\alpha \alpha\rangle$ has a small excess population (depicted by the filled balls), while the uppermost level $|\beta \beta\rangle$ has a slightly depleted population (depicted by the white balls). These population differences are very small in the case of conventional thermal polarization, but may be of the order of 1 in spin hyperpolarization experiments [6-10].

The first pulse sequence may be performed in two versions, $A_{+}$and $A_{-}$, given by the two-pulse sequences $A_{ \pm}=90_{0}-\tau_{1}-90_{\mp 90}$, where the symbol $\beta_{\phi}$ denotes a strong, nonselective pulse with flip angle $\beta$ and phase $\phi$ (both angles are specified in degrees). The delay $\tau_{1}$ is set to the value $\left|\pi / \omega_{\Delta}^{\text {high }}\right|$, where $\omega_{\Delta}^{\text {high }}=-\gamma B_{\text {high }}\left(\delta_{2}-\delta_{1}\right)$. It is readily shown by standard methods [2] that the sequence $A_{+}$exchanges the population of state $|\alpha \alpha\rangle$ with that of $|\alpha \beta\rangle$ and that of $|\beta \alpha\rangle$ with that of $|\beta \beta\rangle$. Hence, at time point (2), a nonequilibrium population distribution is generated, in which level $|\alpha \beta\rangle$ has an excess population, while $|\beta \alpha\rangle$ has a depleted population, as shown in Fig. 1(c). The sequence $A_{-}$may be used instead of $A_{+}$to generate a depleted population of the $|\alpha \beta\rangle$ state at the time point (2). The sequence $A_{-}$exchanges the population of state $|\alpha \alpha\rangle$ with that of $|\beta \alpha\rangle$ and that of $|\alpha \beta\rangle$ with that of $|\beta \beta\rangle$.

During the interval $\tau_{\text {transp }}^{(1)}$, the sample is transported to a region of low magnetic field $B_{\text {low }}$, which is of the order of millitesla. In low field, it is convenient to discuss the behavior of the nuclear spins using the four eigenstates of the pure $J$ coupling Hamiltonian $\mathcal{H}_{J}=2 \pi J I_{1} \cdot I_{2}$. The four $J$ coupling eigenstates may be classified as the three components of a triplet state, plus a singlet state. The triplet state components, denoted $\left|T_{1}\right\rangle=|\alpha \alpha\rangle,\left|T_{0}\right\rangle=$ $(1 / \sqrt{2})(|\alpha \beta\rangle+|\beta \alpha\rangle)$ and $\left|T_{-1}\right\rangle=|\beta \beta\rangle$, have the same $J$ coupling eigenvalue of $+\frac{1}{2} \pi J$. The singlet state, denoted $\left|S_{0}\right\rangle=(1 / \sqrt{2})(|\alpha \beta\rangle-|\beta \alpha\rangle)$, has a $J$ coupling eigenvalue of $-\frac{3}{2} \pi J$. The triplet and singlet states are energy eigenstates of the nuclear spins in zero magnetic field. The energy level diagram in this case is depicted next to time point (3) in Fig. 1(c).

If the transport from high to low field is fast compared to $T_{1}$ but slow compared to the $J$ coupling and if $J \geq 0$, the excess population of the high-field eigenstate $|\alpha \beta\rangle$ is transferred adiabatically into an excess population of the low-field eigenstate $\left|S_{0}\right\rangle$. Similarly, the depleted population of the high-field eigenstate $|\beta \alpha\rangle$ is transferred adiabatically into a depleted population of the zero-field eigenstate $\left|T_{0}\right\rangle$. These adiabatic transfer processes have been exploited before in the context of parahydrogenenhanced NMR [9]. The idealized distribution of the singlet and triplet state populations is shown at time point (3) in Fig. 1(c). This assumes pure adiabatic transfer and neglects $T_{1}$ relaxation during the transport interval.

If the storage field is exactly zero and if intramolecular dipole-dipole coupling provides the only relaxation mechanism, the singlet state population is stable indefinitely, while the populations of the three triplet states rapidly equilibrate. In practice, the LFS state population decays with a long time constant $T_{\mathrm{LFS}}$, due to other relaxation mechanisms and to the small field $B_{\text {low }}$. The expected population distribution after a time $\tau_{\mathrm{LF}}$ in low field, where $T_{\mathrm{LFS}} \approx T_{\mathrm{LF}} \gg T_{1}$, is sketched next to time point (4) in Fig. 1(c). The singlet population may be read out by adiabatic transport of the sample back into high field during the interval $\tau_{\text {transp. }}^{(2)}$ This transforms the LFS population into a population of the high-field $|\alpha \beta\rangle$ state, as depicted for time point (5) in Fig. 1(c). The population of the $|\alpha \beta\rangle$ state is converted into observable NMR signals by the pulse sequence $B$, given by $90_{0}-\tau_{2}-$ $180_{90}-\tau_{3}-90_{45}$, where the delays are given by $\tau_{2}=\left|\pi /\left(2 \omega_{\Delta}^{\text {high }}\right)\right|+|1 /(4 J)|$ and $\tau_{3}=|1 /(4 J)|$. A calculation using standard methods [2] shows that this sequence converts the $|\alpha \beta\rangle$ state population into signal components which provide antiphase NMR signals centered at the chemical shift $\delta_{2}$ after Fourier transformation. NMR signals deriving from $T_{1}$ relaxation during the transport interval, on the other hand, only generate NMR signals at the chemical shift $\delta_{1}$. The interesting NMR signals deriving from LFS storage are therefore cleanly separated from signals with a trivial origin. By repeating the experiment with different values of $\tau_{\mathrm{LF}}$ and observing the behavior of the NMR signals at the chemical shift $\delta_{2}$, it is possible to study the slow decay of the LFS state.

The NMR experiments were performed on a Varian Infinity+ at a field of $B_{\text {high }} \approx 9.4 \mathrm{~T}$, using an actively shielded $89 \mathrm{~mm}$ bore magnet and a standard $5 \mathrm{~mm}$ 
high-resolution NMR probe. The $90^{\circ}$ pulse duration was around $12 \mu \mathrm{s}$. The sample was transported between the high and low fields using the standard pneumatic sample elevator. The air pressure of the elevator was adjusted in order to accelerate the transport times. The transport times $\tau_{\text {transp }}^{(1)}=9 \pm 1 \mathrm{~s}$ and $\tau_{\text {transp }}^{(2)}=13 \pm 2 \mathrm{~s}$, measured from when the sample is fully loaded into the coil to when the sample is outside the magnet bore. The field $B_{\text {low }}$ were estimated using a Hall-effect Gauss meter, with zero-field calibrated to an accuracy of approximately $10 \mu \mathrm{T}$ using a Mumetal shield far from the magnet. For a storage field $B_{\text {low }}=20 \mathrm{mT}$, the sample was elevated to the top of the magnet bore and allowed to remain there for the time $\tau_{\mathrm{LF}}$. For the lower fields, the sample was carried physically to calibrated locations within the stray field of the magnet, or in the case $B_{\text {low }}=0$, into the magnetically shielded chamber.

The sample was $500 \mu \mathrm{l}$ of a $17.7 \mathrm{mM}$ solution of 2,3-dibromothiophene in dimethylsulfoxide- $\mathrm{d}^{6}$ $\left(\right.$ DMSO- $\left.\mathrm{d}^{6}\right)$, contained in a $5 \mathrm{~mm}$ high-resolution NMR tube equipped with a Young valve to facilitate degassing. The sample contained a water impurity in approximately $27 \mathrm{mM}$ concentration and it was subjected to three freezepump-thaw degassing cycles, each lasting over $30 \mathrm{~min}$, to remove dissolved oxygen.

A conventional proton NMR spectrum, obtained at a field $B_{\text {high }} \approx 9.4 \mathrm{~T}$, is shown in Fig. 2(a) and displays the two doublets of a typical $A X$ spin system, with chemical shifts $\delta_{1}=7.11 \mathrm{ppm}, \quad \delta_{2}=7.72 \mathrm{ppm}$, and $J=$ $5.7 \mathrm{~Hz}$. An inversion-recovery series (not shown), shows that the $T_{1}$ time constants for the inequivalent sites are very similar in high field $\left[T_{1}\left(\delta_{1}\right)=16.5 \pm 0.2 \mathrm{~s}\right.$; $\left.T_{1}\left(\delta_{2}\right)=17.1 \pm 0.2 \mathrm{~s}\right]$. Figure $2(\mathrm{~b})$ shows the spectrum obtained after the singlet-storage sequence in Fig. 1, using a storage time $\tau_{\mathrm{LF}}=100 \mathrm{~s}$, and a storage field $B_{\text {low }}=$ $1.8 \pm 0.5 \mathrm{mT}$. The pulse sequence delays were $\tau_{1}=2.05$, $\tau_{2}=44.64$, and $\tau_{3}=43.61 \mathrm{~ms}$. The spectrum in Fig. 2(b) shows diagnostic antiphase signals at around $\delta_{2}=$ $7.72 \mathrm{ppm}$ and an antiphase dispersion structure around $\delta_{1}=7.11 \mathrm{ppm}$. The antiphase signals around $\delta_{2}=$ $7.72 \mathrm{ppm}$ in Fig. 2 are due to long-term storage of nuclear spin order in the LFS state during the delay $\tau_{\mathrm{LF}}$. This may be proved by replacing the sequence $A_{+}$by $A_{-}$, which leads to a change in the sign of the LFS population with respect to equilibrium. Figure 2(c) shows the expected change in sign of the diagnostic antiphase signals around $\delta_{2}=7.72 \mathrm{ppm}$. The signals around $\delta_{1}=7.11 \mathrm{ppm}$ are also perturbed, but in a more complicated way, since these signals are superpositions of several contributions. Since the total interval $\tau_{\text {transp }}^{(1)}+\tau_{\mathrm{LF}}+\tau_{\text {transp }}^{(2)}=120 \mathrm{~s} \mathrm{ex}$ ceeds $T_{1}$ by a factor of 7, the spectra in Figs. 2(b) and 2(c) prove that the memory of the nuclear spin system has been extended significantly beyond the $T_{1}$ limit. Figure 3 shows the measured decay of the singlet population in low field, derived by monitoring the $7.72 \mathrm{ppm}$ antiphase signals as a function of $\tau_{\mathrm{LF}}$. In a field $B_{\text {low }}=1.8 \pm 0.5 \mathrm{mT}$, the time constant for the singlet decay is estimated to be

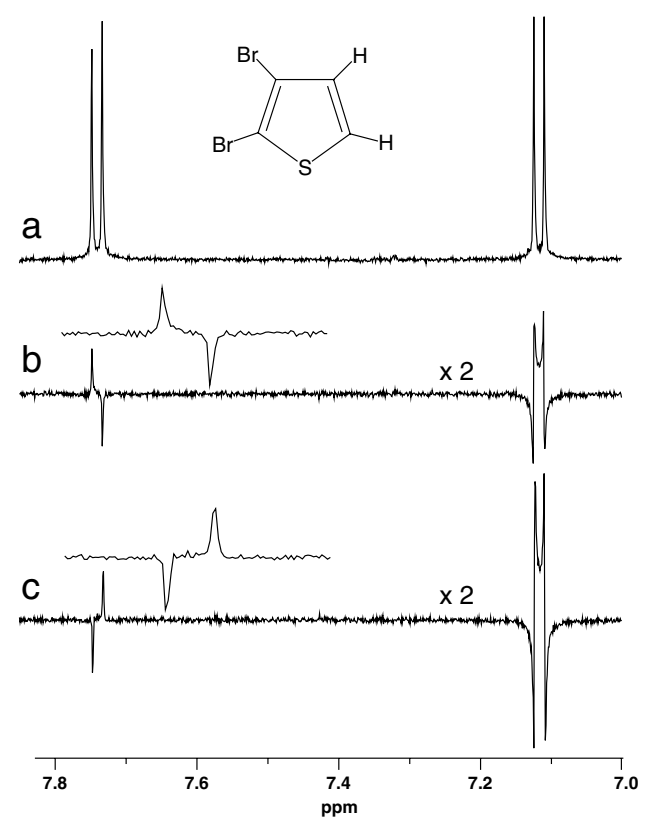

FIG. 2. (a) Conventional ${ }^{1} \mathrm{H}$ NMR spectrum of the solution of 2,3-dibromothiophene (inset) in DMSO-d ${ }^{6}$. (b) Spectrum generated by the sequence in Fig. 1, using the preparation sequence $A_{+}$and a storage time $\tau_{\mathrm{LF}}=100 \mathrm{~s}$. (c) Spectrum generated by the sequence in Fig. 1, using the preparation sequence $A_{-}$and a storage time $\tau_{\mathrm{LF}}=100 \mathrm{~s}$. Expanded sections of the spectra from 7.76 to $7.68 \mathrm{ppm}$ are shown for (b) and (c). All spectra were obtained from a single signal acquisition. The spectra in (b) and (c) are expanded vertically by a factor of 2 compared to (a).

$T_{\mathrm{LFS}}=104 \pm 5 \mathrm{~s}$, with no appreciable field dependence for magnetic fields less than $B_{\text {low }}=20 \mathrm{mT}$. We have analyzed the dynamics of spin pairs in low magnetic field and predict that a significantly enhanced singlet decay will only be observed at fields greater than several hundred $\mathrm{mT}$, for this system. This theory will be presented elsewhere.

The zero-field singlet state may be used as a long-term repository for nuclear spin order, protected from intramolecular dipole-dipole relaxation. A strong spin relaxation mechanism may therefore be "turned off" by a change in the applied magnetic field combined with the exploitation of a special nuclear spin state. This phenomenon should not be confused with the field dependence of $T_{1}$, which is very mild for the sample studied here. Instead of changing $T_{1}$, the low external field suppresses the chemical shift difference and renders the spins magnetically equivalent. Intramolecular dipole-dipole relaxation is symmetric with respect to spin exchange, and cannot interconvert the singlet and triplet states, which have different exchange parity. In effect, the singlet nuclear spin state of 2,3-dibromothiophene is an analogue of parahydrogen, which is also a long-lived nuclear spin singlet, due to its intrinsic magnetic equivalence [8-10].

The storage effect should be observable in systems of larger numbers of spins as long as magnetic equivalence 


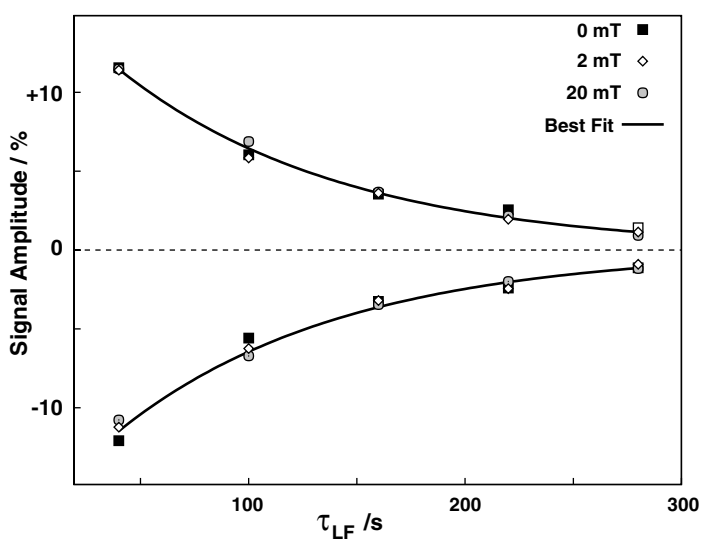

FIG. 3. Decay of the diagnostic antiphase signals as a function of time $\tau_{\mathrm{LF}}$. The top and bottom halves indicate the integrated amplitudes of the two components of the antiphase multiplet centred at $7.72 \mathrm{ppm}$. The symbols represent measurements at different storage fields $B_{\text {low }}$. The vertical scale is the integrated amplitude of the spectral peaks as a percentage of their amplitude in an ordinary one-pulse NMR experiment. The solid line fits to decaying exponential functions with time constant $T_{\mathrm{LFS}}=104 \mathrm{~s}$. The singlet decay is independent of the storage field in this regime.

can be switched on and off by a change in the external magnetic field strength. We are currently exploring fourproton systems of the form $\mathrm{X}-\mathrm{CH}_{2}-\mathrm{CH}_{2}-\mathrm{Y}$ which should display a similar property. A key feature of the current phenomenon is that a new symmetry element of the spin Hamiltonian is introduced by an external agent, in this case the near-removal of the external magnetic field. Analogous effects are expected when the molecular symmetry is switched by, for example, photoisomerization or other chemical reactions. Experiments may be contemplated in which nuclear spin order is trapped, stored for extended periods, and released by magnetic field changes or by light-induced isomerization events.

Singlet storage effects may be observable entirely in high magnetic field if a suitable rf pulse sequence is used to suppress chemical shift differences [17]. Analogous "zero-field experiments in high field" have been performed in solid-state NMR [18,19].

In the system studied here, singlet decay is observed to accelerate when the proton concentration in the solution is increased. This indicates that intermolecular protonproton relaxation is a strong contributor to the singlet relaxation and that very long lifetimes may only be achievable in highly dilute solutions. Singlet relaxation is also expected to be caused by paramagnetic impurities such as dissolved oxygen, intermolecular interactions with solvent deuterons, and scalar relaxation of the second kind [20] via the $\mathrm{Br}$ nuclei. It remains to be seen whether the phenomenon is broadly applicable or whether it is limited to highly specific systems.

Despite these reservations, we feel that the first observation of spin memory extension beyond the $T_{1}$ limit in a system lacking intrinsic magnetic equivalence is suggestive of a new range of NMR experiments, which exploit longer time scales than currently accessible. The long lifetime of LFS states is expected to facilitate nuclear hyperpolarization experiments, especially those involving parahydrogen [8-10], it may create new possibilities in the exploitation of NMR for quantum computation [3,4], for imaging [21], for flow and diffusion studies [22], and for biomolecular structure determination [5].

This research was supported by the EPSRC (U.K.) and the University of Southampton (U.K.). We wish to acknowledge stimulating discussions with Walter Köckenberger, Nottingham University, U.K.

*Electronic address: mhl@ soton.ac.uk

[1] R. R. Ernst, G. Bodenhausen, and A. Wokaun, Principles of Nuclear Magnetic Resonance in One and Two Dimensions (Clarendon Press, Oxford, 1988).

[2] M. H. Levitt, Spin Dynamics. Basics of Nuclear Magnetic Resonance (Wiley, Chichester, United Kingdom, 2001).

[3] N. A. Gershenfeld and I. L. Chuang, Science 275, 350 (1997).

[4] D. G. Cory, A. F. Fahmy, and T. F. Havel, Proc. Nat. Acad. Sci. U.S.A. 94, 1634 (1997).

[5] K. Wüthrich, Nat. Struct. Biol. 5, 492 (1998).

[6] G. Navon, Y.-Q. Song, T. Room, S. Appelt, R. E. Taylor, and A. Pines, Science 271, 1848 (1996).

[7] D. A. Hall, D. C. Maus, G. J. Gerfen, S. J. Inati, L. R. Becerra, F.W. Dahlquist, and R. G. Griffin, Science 276, 930 (1997).

[8] C. R. Bowers and D. P. Weitekamp, J. Am. Chem. Soc. 109, 5541 (1987).

[9] M. G. Pravica and D. P. Weitekamp, Chem. Phys. Lett. 145, 255 (1988).

[10] K. Golman, O. Axelsson, H. Johannesson, S. Månsson, C. Olofsson, and J. S. Petersson, Magn. Reson. Med. 46, 1 (2001).

[11] D. A. Lidar, I. L. Chuang, and K. B. Whaley, Phys. Rev. Lett. 81, 2594 (1998).

[12] L. Viola, E. M. Fortunato, M. A. Pravia, E. Knill, R. Laflamme, and D. G. Cory, Science 293, 2059 (2001).

[13] E. M. Fortunato, L. Viola, J. Hodges, G. Teklemariam, and D. G. Cory, New J. Phys. 4, 5 (2002).

[14] L. Viola, E. Knill, and S. Lloyd, Phys. Rev. Lett. 85, 3520 (2000).

[15] P. Zanardi, Phys. Rev. A 63, 012301 (2001).

[16] D. A. Lidar and K. B. Whaley, Irreversible Quantum Dynamics, Lecture Notes in Physics Vol. 622 (Springer, Berlin, 2003), pp. 83-120.

[17] J. D. Ellett and J.S. Waugh, J. Chem. Phys. 51, 2851 (1969).

[18] R. Tycko, Phys. Rev. Lett. 60, 2734 (1988).

[19] R. Tycko, J. Chem. Phys. 92, 5776 (1990).

[20] A. Abragam, The Principles of Nuclear Magnetism (Clarendon Press, Oxford, 1961).

[21] M. T. Vlaardingerbroek, J. A. de Boer, and A. Luiten, Magnetic Resonance Imaging: Theory and Practice (Springer-Verlag, Berlin, 2002).

[22] P.T. Callaghan, Rep. Prog. Phys. 62, 599 (1999). 